\title{
COMMENT
}

ECOLOGY What motivated Karl von Frisch to unravel bee communication? p.32
TECHNOLOGY The evolution of artificial intelligence, its potential and pitfalls p.33
PHysics Sean Carroll on quantum field theory and morality p.34

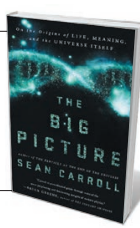

CONSERVATION Anchors are another way that shipping fouls the environment p.36

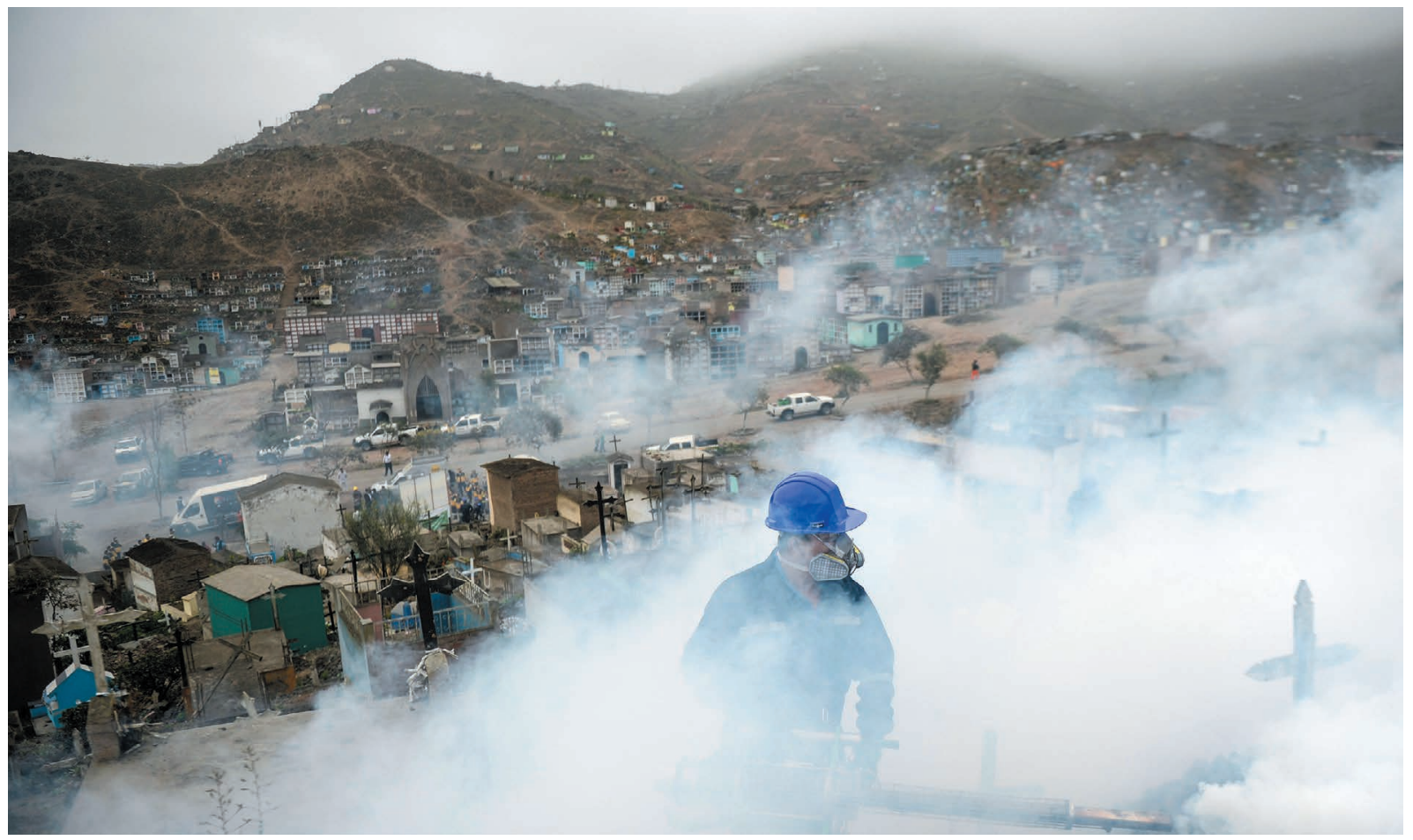

Specialists in Peru fumigate a cemetery in an effort to prevent Chikungunya and Zika viruses from spreading.

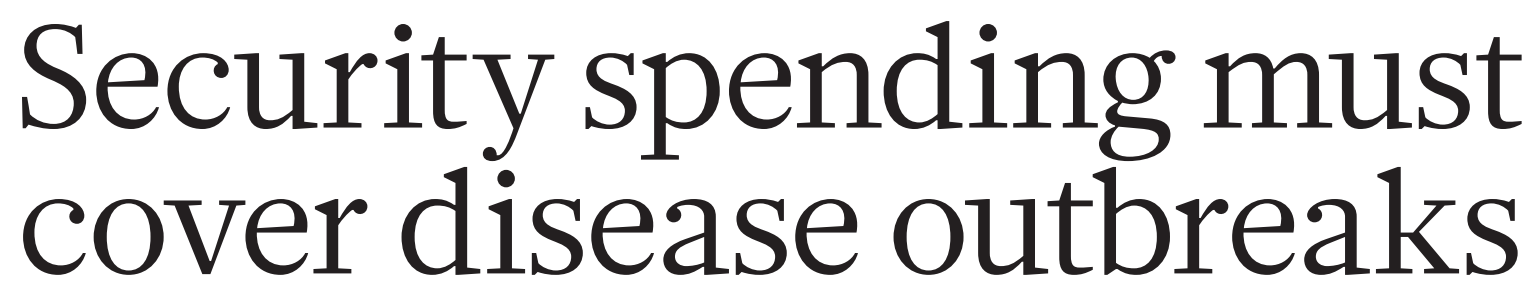

Tadataka Yamada, V. Ayano Ogawa and Maria Freire call for research and development funding and coordination to counter global infectious-disease threats.

$\mathrm{T}$ The health emergency precipitated by the Zika virus is a salutary reminder: global preparedness for emerging pathogens with endemic or pandemic potential is crucial and needs an overhaul. These crises are not rare - Lassa fever, Ebola virus, Middle East respiratory syndrome, H1N1 influenza and severe acute respiratory syndrome (SARS) have surfaced in head-spinning succession over the past 10-15 years. Each emergence proves how woefully unprepared the global community is to deal with worldwide health emergencies that have deep societal and economic impact.
Diagnostic tools, medicines and vaccines are in limited supply, non-existent or too costly - many people die and many more suffer in each outbreak as a result. Fear and panic spread, borders are closed, travel is restricted and commerce is shut down. After the recent Ebola outbreak in West Africa, the direct financial repercussions on Liberia, Sierra Leone and Guinea could amount to around $10 \%{ }^{1}$ of the nations' gross domestic product for 2014-15; the cost of SARS to the global economy in 2003, exceeded US\$40 billion ${ }^{2}$.

The health, economic and social consequences of a global health emergency are as great a threat to global and nationa security as those of terrorist actions. Although the world has gone to great expense and effort to prepare for the latter, it has done unacceptably little to prepare for the former, given the solemn responsibility of nations to ensure the health and security of their citizens. The United States spends at least $\$ 100$ billion a year on counterterrorism efforts; it invests just $\$ 1$ billion on pandemic and emerging infectious-disease programmes ${ }^{3}$.

In this context, the Commission on 
a Global Health Risk Framework for the Future - an independent, international panel - published recommendations in January for addressing future global infectious-disease threats ${ }^{4}$. The 17-member commission has a secretariat at the US National Academy of Medicine. It was supported by seven private donors as well as the US Agency for International Development, and sought advice from more than 200 global technical experts from government, private industry, academia, non-governmental organizations and foundations. The commission's report addressed pandemic preparedness from four perspectives: governance, health systems, financing and research and development (R\&D).

Here we expand on the R\&D element of these recommendations. Several excellent global proposals and initiatives have arisen in the past year that are relevant to R\&D for pandemic preparedness. One is a proposal to create a fund to support vaccine development. Another is an R\&D Blueprint, issued by the World Health Organization (WHO), which aims to implement a road map for R\&D preparedness for known priority pathogens and to facilitate roll out of an emergency $R \& D$ response in a timely manner for emerging ones. But gaps remain - conceptually, practically and financially - and these need to be plugged, urgently, in the following ways.

\section{MORE FUNDS}

Society - national governments, industry, charities and others - needs to invest an extra $\$ 1$ billion per year for 15 years, over and above the amount currently being spent on R\&D for infectious diseases and global preparedness. This is equivalent to the $R \& D$ budget of a medium-sized pharmaceutical company with a portfolio of products in various stage of development (see go.nature. com/4hfdrj).

These funds would be used in three ways: in the targeted expansion or acceleration of ongoing R\&D projects (excluding those that address antimicrobial resistance, which deserve their own targeted funds and efforts); for the development of core functions, such as clinical-trial infrastructure and manufacturing capacity; and to spur innovation, especially in new platforms that could allow 'plug and play' strategies, offering the potential to move quickly from the identification of a pathogen to the development and manufacturing of a product.

Is $\$ 1$ billion too much or too little? It is less than $2 \%$ of the United States' annual budget for homeland security ${ }^{5}$ and less than $0.2 \%$ of its defence budget ${ }^{6}$. Thus it is in our view a reasonable, attainable sum. Some feel that there is little enthusiasm from funders, including governments, for extra pooled resources for R\&D - but jump-starting

the enterprise is paramount. The goal is the security of the world's population.

We contend that the money should come from multiple sources, including national-security and defence budgets. In non-emergency times, governments must support the training of scientific and medical personnel to carry out basic-research activities and provide them with adequate local laboratories in which to work. Poorer countries have smaller budgets, but health should be their top priority. This strategy is akin to basic military preparedness, requiring resources, practice, vigilance and longterm commitment.

Other crucial contributors to preparedness include private industry, particularly pharmaceutical and biotechnology companies, foundations, charities and, importantly, non-traditional actors such as insurance companies and other funders. WHO director-general Margaret Chan has noted that the pharmaceutical industry spent almost $\$ 1$ billion to develop Ebola vaccines in the past two years without any return on investment ${ }^{7}$.

To attract and retain more private-sector involvement in R\&D, national governments and foundations must put in place reasonable incentives.

This is key for conditions with uncertain markets or low financial returns. One such lever is the priority-review voucher that may

\section{"We must not repeat the events of the H1N1 influenza pandemic in 2009."}

be issued in the United States by the Food and Drug Administration to those who develop treatments for diseases that typically do not command big commercial markets, such as river blindness (onchocerciasis).

In recent years, philanthropic foundations have played an increasingly important part in $\mathrm{R} \& \mathrm{D}$ for global health. Organizations such as the Bill \& Melinda Gates Foundation and Médecins Sans Frontières (also known as Doctors Without Borders) have funded new mechanisms for drug and vaccine development for conditions including tuberculosis, malaria, dengue fever, leishmaniasis and Chagas disease. Such operations, known as product development partnerships (PDPs), decouple basic-research expenditures and the cost of failure. PDPs are a powerful mechanism to address product gaps, provided that the basic biology of disease is understood and a path for development is identified.

\section{MORE COORDINATION}

To build and expand on independent public and private-sector activities and ensure synergy, we propose the creation of an independent high-level expert committee. It would help to coordinate research activities, prioritize investments, monitor progress, minimize duplication of effort and make timely decisions. This 15-member Pandemic Product Development Committee (PPDC) would help to make the best of scarce new resources, such as the ability to carry out clinical trials on the ground. It would not undertake direct management of any specific project or have decision-making authority over activities and budgets in ongoing research efforts.

The chair of this committee would be appointed by the WHO director-general following broad consultation with the key stakeholders. The chair and the members of the committee, who would be supported by a small, expert secretariat at the WHO, must have extensive knowledge and experience in the discovery, development, regulatory review and manufacture of medical products and related technologies. The PPDC should feature representatives from industry, academia, the civil service and society. The chair would be a standing member of and accountable to an independent technical governing board, proposed by the commission to oversee the global pandemic preparedness effort ${ }^{4}$.

This governance model has ties to, but is separate from, the WHO. The proposal is based on several factors, including the WHO's global responsibility for health emergencies, the need to tap multiple R\&D parties and the importance of providing the highest level of technical expertise in a neutral forum. Making the PPDC fully part of a United Nations agency would limit the flexibility required for rapid decision-making. Divorcing the PPDC completely from the WHO would undermine the agency's leadership role in health emergencies.

\section{MORE ENGAGEMENT}

During a crisis there is an understandable urge to try unproven technologies on people who are certain to die unless something is done. Yet it is only by maintaining a commitment to scientific rigour that the world has medicines that cure, and vaccines that prevent, disease. Efforts to create new treatments, including those for infectious diseases, must include randomized clinical trials despite the challenges, unless there is some other scientifically valid approach that could lead to similarly actionable information.

Under these circumstances, it is evident that the communities in which trials are being conducted and where resultant products will be distributed must be involved in any R\&D effort from the start. Only by understanding their role as partners in the research effort and the societal benefit of their participation in a placebocontrolled trial will clinical-trial volunteers be able to understand and accept 


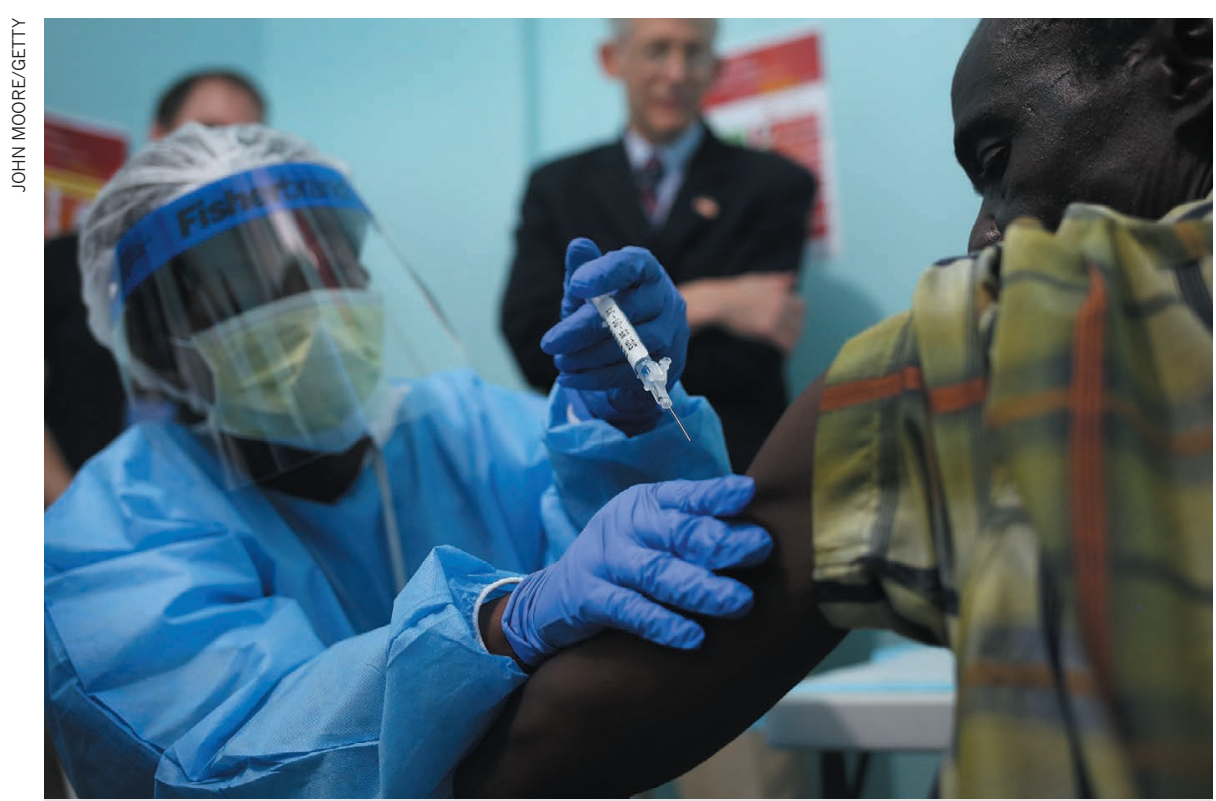

Vaccine trials were conducted during West Africa's devastating Ebola outbreak.

\section{PANDEMIC PREPAREDNESS}

\section{Six steps}

\begin{abstract}
- Negotiate trial designs, including protocols for clinically testing different products against one control group. - Agree sharing policies for reagents, data, patents and other intellectual property.

- Agree regulatory policy, including standards for reviewing and approving products for emergencies, and roles between and within drug agencies in affected countries.
\end{abstract}

- Design liability protection for those who conduct the research and development and for compensation to people affected by unexpected events resulting from experimental interventions.

\section{- Prioritize allocation of resources such} as candidate compounds, instrumentation or clinical-trial sites.

- Ensure capacity for rapid manufacturing, strategic stockpiling and prompt delivery of products. the risks. The communication of crucial information regarding clinical studies will often require the engagement of trusted community or religious leaders and translation into native languages to establish understanding and trust.

Before a crisis, it is the responsibility of all those involved in infectious-disease research and development - public and private - to ensure that drug and vaccine candidates can quickly move forwards. Preparedness must encompass six key activities (see 'Six steps') which should be discussed by the PPDC and implemented by the appropriate stakeholders.

Crucially, stakeholders must agree that the fruits of these efforts will be distributed first to those in greatest need or at greatest risk. We must not repeat the events of the H1N1 influenza pandemic in 2009. Nations with manufacturing facilities distributed vaccines domestically before exporting them; some wealthy nations without vaccine-manufacturing capacity paid substantial sums to reserve the remaining supply. Meanwhile, robust modelling studies indicated that more than $90 \%$ of the deaths from a potential influenza pandemic would probably occur in the world's poorest countries ${ }^{8}$.

\section{ACT NOW}

R\&D for products to address emerging health threats is severely limited and fragmented. Substantial investment and a global commitment are needed to better coordinate independent activities. Components of the basic arsenal such as fit-for-purpose medicines, vaccines, diagnostics and personal protective equipment must exist so that first responders and medical personnel can identify, treat and contain an outbreak.

At the global level, countries must ensure a coordinated, nimble $\mathrm{R} \& \mathrm{D}$ response to health outbreaks. This should include: the comprehensive search for and assessment of existing technologies to tackle the disease; the testing of candidate drugs and vaccines that can be put quickly into development; the repurposing of existing technologies; and worldwide manufacturing capacity that is ready for the rapid production of high-quality drugs and vaccines.

To be clear, the funds we call for are to increase the current worldwide R\&D expenditures, not to replace them. Naturally, basic research into the aetiology of disease and the biology that underpins diseases with pandemic potential must be strongly supported by governments, industry and foundations. Such work is the foundation on which new life-saving tools will be built.

Three principles should guide R\&D for epidemic or pandemic disaster preparedness. First, we must maintain consistently high ethical and scientific standards, particularly during crises. Second, we must define protocols and approaches to engage local scientists and community members early in the conduct of research. And third, we must agree on ways to expedite medical-product approval, manufacture and distribution.

It is imperative that these recommendations are adopted on a global scale. There will be many reasons why some may argue with one or more, and there may be a temptation to delay or forgo the necessary commitments. But we must act. We cannot afford to lose this battle.

Tadataka Yamada is a commissioner on the Global Health Risk Framework Commission, and venture partner at Frazier Healthcare Partners, Seattle, Washington, USA.

V. Ayano Ogawa is associate programme officer for the Global Health Risk Framework Commission at the US National Academy of Medicine, Washington DC, USA. Maria Freire is a commissioner on the Global Health Risk Framework Commission, and president and executive director of the Foundation for the National Institutes of Health, Bethesda, Maryland, USA. e-mail:tachi.yamada@gmail.com

1. United Nations Development Group, Western and Central Africa. Socio-Economic Impact of Ebola Virus Disease in West African Countries: A Call for National and Regional Containment, Recovery and Prevention (United Nations Development Group, 2015).

2. Lee, J. W. \& McKibbin, W. J. in Learning from SARS: Preparing for the Next Disease Outbreak: Workshop Summary (eds Knobler, S. et al.) 92-109 (Natl Acad. Press, 2008).

3. Boddie, C., Sell, T. K. \& Watson, M. Health Secur. 13, 186-206 (2015).

4. Commission on a Global Health Risk Framework for the Future. The Neglected Dimension of Global Security: A Framework to Counter Infectious Disease Crises (Natl Acad. Press, 2016).

5. US Department of Homeland Security. Budgetin-Brief (2015).

6. Office of the Under Secretary of Defense (Comptroller) Chief Financial Officer. United States Department of Defense Fiscal Year 2016 Budget Request: Overview (2015); available at http://go.nature.com/vxnlp4

7. Ward, A. 'WHO chief wants help for drug industry to fight global pandemics' Financial Times (2016).

8. Murray, C. J. L., Lopez, A. D., Chin, B., Feehan, D. \& Hill, K. H. Lancet 368, 2211-2218 (2006).

T.Y. declares competing financial interests: see go.nature.com/chvdjg for details. 